\title{
Split equality problem for $k$-asymptotically strictly pseudo-nonspreading mapping in Hilbert space
}

\author{
Ying Chen ${ }^{\mathrm{a}, \mathrm{b}, *}$, Haili Guo ${ }^{\mathrm{c}}$, Luoyi Shic ${ }^{\mathrm{c}}$, Zhaojun Wang ${ }^{\mathrm{a}}$ \\ a Statistical Research Institute, Naikai University, Tianjin, China. \\ ${ }^{b}$ Tianjin University of Technology and education, Tianjin, 300222, China. \\ ${ }^{c}$ Department of Mathematics, Tianjin Polytechnic University, Tianjin, 300387, China.
}

Communicated by C. Zaharia

\begin{abstract}
In this paper, we consider the split equality problem (SEP) in Hilbert space. We propose and investigate a new iterative algorithm for solving split equality problem for k-asymptotically strictly pseudo-nonspreading mapping. Finally, a numerical example is given to illustrate the feasibility of the proposed algorithm. (C)2017 All rights reserved.
\end{abstract}

Keywords: General split equality problem, k-asymptotically strictly pseudo-nonspreading mapping. 2010 MSC: 47J25, 47H09, 65J25.

\section{Introduction}

In the present paper, we are concerned with the split equality problem (SEP). SEP was proposed by Moudafi in [8]. Let $\mathrm{H}_{1}, \mathrm{H}_{2}$ and $\mathrm{H}_{3}$ be three real Hilbert spaces. Let $\mathrm{T}_{i}: \mathrm{H}_{1} \rightarrow \mathrm{H}_{1}$ be a $\mathrm{k}$-asymptotically strictly pseudo-nonspreading mapping. Denote by $\operatorname{Fix}\left(T_{i}\right)$ the set of fixed points of $T_{i}(i=1,2, \cdots, m)$. Set $\mathrm{C}=\bigcap_{i=1}^{m} \operatorname{Fix}\left(\mathrm{T}_{i}\right)$. Let $A: \mathrm{H}_{1} \rightarrow \mathrm{H}_{3}$ and $\mathrm{B}: \mathrm{H}_{2} \rightarrow \mathrm{H}_{3}$ be two bounded linear operators. Let $\mathrm{Q}$ be a nonempty closed convex subset of $\mathrm{H}_{2}$.

The so-called SEP can mathematically be formulated as

$$
\text { finding } x \in C, y \in Q \text { such that } A x=B y \text {. }
$$

We use $\Gamma$ to denote the solution set of SEP, that is

$$
\Gamma=\left\{(x, y) \in \mathrm{H}_{1} \times \mathrm{H}_{2}, \mathrm{Ax}=\mathrm{By}, \mathrm{x} \in \mathrm{C}, \mathrm{y} \in \mathrm{Q}\right\} .
$$

When $B=I$ (the identity mapping on Hilbert space $H$ ), problem (1.1) is equivalent to the well-known split feasibility problem (SFP).

As we have known, the SEP has received much attention due to its application in various disciplines such as medical image reconstruction and radiation therapy treatment planning [3,4].

\footnotetext{
*Corresponding author

Email addresses: 18630852201@163.com (Ying Chen), 1101570027@qq.com (Haili Guo), shiluoyi@tjpu.edu.cn (Luoyi Shi), zjwang@nankai.edu.cn (Zhaojun Wang)

doi:10.22436/jnsa.010.11.22
} 
To solve the SEP, Moudafi [7, 8] put forward an alternating CQ-algorithm and the relaxed alternating CQ-algorithm. In this paper, inspired by Chang [9], we propose and investigate a new iterative algorithm for solving split equality problem for $\mathrm{K}$-asymptotically strictly pseudo-nonspreading mapping and show the convergence of the presented algorithm. At last we give a numerical example for SEP in $R^{2}$.

\section{Preliminaries}

We recall some definitions, notations and conclusions which will be used in proving our main result. Let $\mathrm{H}$ be a real Hilbert space with inner product $\langle\cdot, \cdot\rangle$ and the norm $\|\cdot\|$. We write $x_{n} \rightarrow x$ (respectively, $x_{n} \rightarrow x$ ), the strong (respectively weak) convergence of the sequence $\left\{x_{n}\right\}$ to $x$.

Let $E$ be a Banach space. A mapping $T$ with domain $D(T)$ in $E$ is said to be demi-closed, if for any sequence $x_{n} \subset E, x_{n} \rightarrow x^{*} \in D(T)$ and $\left\|x_{n}-T x_{n}\right\| \rightarrow 0$, then $T x^{*}=x^{*}$.

A Banach space $E$ is said to have the Opial property, if for any sequence $\left\{x_{n}\right\}$ with $x_{n} \rightarrow x^{*}$, we have

$$
\liminf _{n \rightarrow \infty}\left\|x_{n}-x^{*}\right\|<\liminf _{n \rightarrow \infty}\left\|x_{n}-y\right\|
$$

for all $y \in E$ with $y \neq x^{*}$.

Remark 2.1. It is known that each Hilbert space possesses the Opial property.

Definition 2.2. Let $\mathrm{H}$ be a Hilbert space and $\mathrm{K}$ be a nonempty closed convex subset of $\mathrm{H}$. We denote by Fix $(T)$ the fixed points set of $T$. $T$ is said to be

(i) Nonexpasive, if $\|T x-T y\| \leqslant\|x-y\|$ for all $x, y \in K$.

(ii) $k$-strictly pseudo-nonspreading [2], if there exists $k \in[0,1)$ such that

$$
\|T x-T y\|^{2} \leqslant\|x-y\|^{2}+k\|x-T x-(y-T y)\|^{2}+2\langle x-T x, y-T y\rangle
$$

for all $x, y \in D(T)$.

(iii) $k$-asymptotically strictly pseudo-contraction [5], if there exists a constant $k \in[0,1)$ and a sequence $k_{n} \geqslant 1$ and $\lim _{n \rightarrow \infty} k_{n}=1$ such that

$$
\left\|T^{n} x-T^{n} y\right\|^{2} \leqslant k_{n}\|x-y\|^{2}+k\left\|x-T^{n} x-\left(y-T^{n} y\right)\right\|^{2}
$$

for all $x, y \in D(T)$.

(iv) $k$-asymptotically strictly pseudo-nonspreading [9], if there exists a constant $k \in[0,1)$ and a sequence $k_{n} \geqslant 1$ and $\lim _{n \rightarrow \infty} k_{n}=1$, such that

$$
\left\|T^{n} x-T^{n} y\right\|^{2} \leqslant k_{n}\|x-y\|^{2}+k\left\|x-T^{n} x-\left(y-T^{n} y\right)\right\|^{2}+2\left\langle x-T^{n} x, y-T^{n} y\right\rangle
$$

for all $x, y \in D(T)$.

Remark 2.3. $\mathrm{k}$-asymptotically strictly pseudo-nonspreading is much more general than $\mathrm{k}$-strictly pseudononspreading and $\mathrm{k}$-asymptotically strictly pseudo-contraction.

For every point $x \in H$, there exists a unique point $P_{K} x \in K$, such that

$$
\left\|x-P_{K} x\right\| \leqslant\|x-y\|, \forall y \in K,
$$

where $P_{K}$ is called the metric projection of $H$ onto $K$. We know that $P_{K}$ is a nonexpansive mapping of $H$ onto $\mathrm{K}$.

Lemma 2.4 ([9]). Let $\mathrm{K}$ be a nonempty closed convex subset of a real Hilbert space $\mathrm{H}$, and let $\mathrm{T}: \mathrm{K} \rightarrow \mathrm{K}$ be a continuous $\mathrm{K}$-asymptotically strictly pseudo-nonspreading. If $\operatorname{Fix}(\mathrm{T})$ is nonempty, then it is a closed and convex subset. 
Lemma 2.5 ([1]). Let $\left\{a_{n}\right\}_{n=0}^{\infty},\left\{b_{n}\right\}_{n=0}^{\infty}$ be sequences of nonnegative numbers satisfying $a_{n+1} \leqslant a_{n}+b_{n}$, for all $\mathrm{n} \geqslant 0$. If $\sum_{n=0}^{\infty} b_{n}<\infty$, then $\lim _{n \rightarrow \infty} a_{n}$ exists.

Lemma 2.6 ([6]). We use $\Gamma$ to denote the solution set of SEP, that is

$$
\Gamma=\left\{(x, y) \in \mathrm{H}_{1} \times \mathrm{H}_{2}, \mathrm{Ax}=\mathrm{By}, \mathrm{x} \in \mathrm{C}, \mathrm{y} \in \mathrm{Q}\right\},
$$

and assume consistency of SEP, so that $\Gamma$ is nonempty closed convex.

Let $\mathrm{S}=\mathrm{C} \times \mathrm{Q}, \mathrm{C}$ and $\mathrm{Q}$ be two nonempty closed convex subsets of real Hilbert spaces $\mathrm{H}_{1}$ and $\mathrm{H}_{2}$, define

$$
\mathrm{G}=\left[\begin{array}{ll}
\mathrm{A} & -\mathrm{B}
\end{array}\right]
$$

$\mathrm{G}: \mathrm{H} \rightarrow \mathrm{H}_{3}$, then

$$
G^{*} G=\left[\begin{array}{cc}
A^{*} A & -A^{*} B \\
-B^{*} A & B^{*} B
\end{array}\right]
$$

The original problem can be reformulated as

$$
\text { finding } w=\left[\begin{array}{l}
x \\
y
\end{array}\right] \text { with } \quad G w=0
$$

Then, $w=\left[\begin{array}{l}x \\ y\end{array}\right]$ solves the SEP if and only if $w$ solves the fixed point equation

$$
P_{S}\left(w-\gamma G^{*} G w\right)=w .
$$

Lemma 2.7 ([10]). Let $J=I-\gamma G^{*} G$, where $0<\gamma<2 / \rho\left(G^{*} G\right), \rho\left(G^{*} G\right)$ being the spectral radius of the self-adjoint operator $\mathrm{G}^{*} \mathrm{G}$ on $\mathrm{H}$. Then we have the following property:

$$
\begin{aligned}
\operatorname{Fix}(J) & =\{(x, y) \in H, A x=B y\}, \\
\operatorname{Fix}\left(P_{S} J\right) & =\operatorname{Fix}\left(P_{S}\right) \bigcap \operatorname{Fix}(J)=\Gamma .
\end{aligned}
$$

\section{Main result}

Theorem 3.1. Let $\mathrm{H}_{1}$ and $\mathrm{H}_{2}$ be two real Hilbert spaces. Let $\mathrm{T}_{i}: \mathrm{H}_{1} \rightarrow \mathrm{H}_{1}$ be a $\mathrm{k}$-asymptotically strictly pseudononspreading mapping. Let $\mathrm{C}=\bigcap_{i=1}^{m} \operatorname{Fix}\left(\mathrm{T}_{i}\right)$ and $\mathrm{Q}$ be any nonempty closed convex set of $\mathrm{H}_{2}$. Let $\mathrm{S}=\mathrm{C} \times \mathrm{Q}$ and $\mathrm{P}_{\mathrm{S}}$ be the metric projection of $\mathrm{H}=\mathrm{H}_{1} \times \mathrm{H}_{2}$ to $\mathrm{S}$. Let $\left\{w_{\mathrm{n}}\right\}$ be generated by

$$
\left\{\begin{array}{l}
w_{1} \in H, \\
u_{n}=\left(I-\gamma G^{*} G\right) w_{n}, \\
w_{n+1}=P_{S}\left[\left(1-\alpha_{n}\right) u_{n}+\alpha_{n} T_{[n]}^{n} u_{n}\right],
\end{array}\right.
$$

where $[n]=n \bmod m, 0<\gamma<\lambda=2 / \rho\left(G^{*} G\right)$, and $\rho\left(G^{*} G\right)$ being the spectral radius of the self-adjoint operator $\mathrm{G}^{*} \mathrm{G},\left\{\alpha_{n}\right\}$ is a sequence in $(0,1-\mathrm{k}]$, and $\sum_{n=0}^{\infty} \alpha_{n}<\infty$ and we remember $T_{i}$ as $T_{i} \oplus \mathrm{I}$. If $\Gamma$ is nonempty, then the sequence $w_{n}$ converges weakly to a point $w \in \Gamma$.

Proof. The proof is divided into five steps.

(1) We first prove the $\lim _{n \rightarrow \infty}\left\|w_{n}-p\right\|$ exists, for any $p \in \Gamma$.

Since $p \in \Gamma$, we have $p \in \bigcap_{i=1}^{m} \operatorname{Fix}\left(T_{i}\right)$ and $p \in \operatorname{Fix}\left(P_{S} J\right)$. It follows from (3.1) that

$$
\begin{aligned}
\left\|w_{n+1}-p\right\|^{2} & =\left\|P_{S}\left[\left(1-\alpha_{n}\right) u_{n}+\alpha_{n} T_{[n]}^{n} u_{n}\right]-P_{S} p\right\|^{2} \\
& \leqslant\left\|u_{n}-p+\alpha_{n}\left(T_{[n]}^{n} u_{n}-u_{n}\right)\right\|^{2} \\
& =\left\|u_{n}-p\right\|^{2}+2 \alpha_{n}\left\langle u_{n}-p, T_{[n]}^{n} u_{n}-u_{n}\right\rangle+\alpha_{n}^{2}\left\|T_{[n]}^{n} u_{n}-u_{n}\right\|^{2} .
\end{aligned}
$$


Because $T_{i}$ is a $k$-asymptotically strictly pseudo-nonspreading for any $v \in H$, we have

$$
\left\|T_{[n]}^{n} u_{n}-T_{[n]}^{n} v\right\|^{2} \leqslant k_{n}\left\|u_{n}-v\right\|^{2}+2\left\langle T_{[n]}^{n} u_{n}-u_{n}, v-T_{[n]}^{n} v\right\rangle+k\left\|T_{[n]}^{n} u_{n}-u_{n}-\left(v-T_{[n]}^{n} v\right)\right\|^{2} .
$$

Taking $v=p$, we have

$$
\left\|T_{[n]}^{n} u_{n}-p\right\|^{2} \leqslant k_{n}\left\|u_{n}-p\right\|^{2}+k\left\|T_{[n]}^{n} u_{n}-u_{n}\right\|^{2}
$$

Observe that

$$
\begin{aligned}
\left\|T_{[n]}^{n} u_{n}-p\right\|^{2} & =\left\|T_{[n]}^{n} u_{n}-u_{n}+u_{n}-p\right\|^{2} \\
& =\left\|T_{[n]}^{n} u_{n}-u_{n}\right\|^{2}+2\left\langle T_{[n]}^{n} u_{n}-u_{n}, u_{n}-p\right\rangle+\left\|u_{n}-p\right\|^{2} \\
& \leqslant k_{n}\left\|u_{n}-p\right\|^{2}+k\left\|T_{[n]}^{n} u_{n}-u_{n}\right\|^{2} .
\end{aligned}
$$

Simplify the above inequality, we have

$$
2 \alpha_{n}\left\langle T_{[n]}^{n} u_{n}-u_{n}, u_{n}-p\right\rangle \leqslant \alpha_{n}(k-1)\left\|T_{[n]}^{n} u_{n}-u_{n}\right\|^{2}+\alpha_{n}\left(k_{n}-1\right)\left\|u_{n}-p\right\|^{2} .
$$

It follows from (3.2) and (3.3) that

$$
\begin{aligned}
\left\|w_{n+1}-p\right\|^{2} & \leqslant\left(\alpha_{n}\left(k_{n}-1\right)+1\right)\left\|u_{n}-p\right\|^{2}+\alpha_{n}^{2}\left\|T_{[n]}^{n} u_{n}-u_{n}\right\|^{2}+\alpha_{n}(k-1)\left\|T_{[n]}^{n} u_{n}-u_{n}\right\|^{2} \\
& =\left(\alpha_{n}\left(k_{n}-1\right)+1\right)\left\|u_{n}-p\right\|^{2}-\alpha_{n}\left(1-k-\alpha_{n}\right)\left\|T_{[n]}^{n} u_{n}-u_{n}\right\|^{2} .
\end{aligned}
$$

On the other hand,

$$
\begin{aligned}
& \left\|u_{n}-p\right\|^{2}=\left\|\left(I-\gamma G^{*} G\right) w_{n}-p\right\|^{2} \\
& =\left\|w_{n}-p-\gamma G^{*} \mathrm{G} w_{n}\right\|^{2} \\
& =\left\|w_{n}-p\right\|^{2}-2 \gamma\left\langle w_{n}-p, G^{*} G w_{n}\right\rangle+\gamma^{2}\left\|G^{*} G w_{n}\right\|^{2} \\
& =\left\|w_{n}-p\right\|^{2}-2 \gamma\left\langle\mathrm{G} w_{n}-\mathrm{Gp}, \mathrm{G} w_{n}\right\rangle+\gamma^{2}\left\langle\mathrm{G}^{*} \mathrm{G} w_{n}, \mathrm{G}^{*} \mathrm{G} w_{n}\right\rangle \\
& =\left\|w_{n}-p\right\|^{2}-2 \gamma\left\langle G w_{n}-G p, G w_{n}\right\rangle+\gamma^{2}\left\langle G w_{n}, G G^{*} G w_{n}\right\rangle \\
& \leqslant\left\|w_{n}-p\right\|^{2}-2 \gamma\left\|\mathrm{G} w_{n}\right\|^{2}+\left(2 \gamma^{2} / \lambda\right)\left\|\mathrm{G} w_{n}\right\|^{2} \\
& =\left\|w_{n}-p\right\|^{2}-\gamma(2-2 \gamma / \lambda)\left\|G w_{n}\right\|^{2} \text {. }
\end{aligned}
$$

It follows from (3.4) and (3.5) that

$$
\begin{aligned}
\left\|w_{n+1}-p\right\|^{2} \leqslant & \left(\alpha_{n}\left(k_{n}-1\right)+1\right)\left\|w_{n}-p\right\|^{2}-\gamma\left(\alpha_{n}\left(k_{n}-1\right)+1\right)(2-2 \gamma / \lambda)\left\|G w_{n}\right\|^{2} \\
& -\alpha_{n}\left(1-\kappa-\alpha_{n}\right)\left\|T_{[n]}^{n} u_{n}-u_{n}\right\|^{2} \\
\leqslant & \left(\alpha_{n}\left(k_{n}-1\right)+1\right)\left\|w_{n}-p\right\|^{2} .
\end{aligned}
$$

Put $x_{n}=\left\|w_{n}-p\right\|^{2}$ and $\beta_{n}=\alpha_{n}\left(k_{n}-1\right)$, then (3.6) is equivalent to

$$
x_{n+1} \leqslant\left(\beta_{n}+1\right) x_{n}=x_{n}+\beta_{n} x_{n} .
$$

By (3.7), we know

$$
\begin{aligned}
x_{n+1} & \leqslant\left(1+\beta_{n}\right) x_{n} \\
& \leqslant\left(1+\beta_{n}\right)\left(1+\beta_{n-1}\right) \cdots\left(1+\beta_{0}\right) x_{0} \\
& =e^{\ln \left(1+\beta_{n}\right)+\ln \left(1+\beta_{n-1}\right)+\cdots+\ln \left(1+\beta_{0}\right)} \chi_{0} \\
& \leqslant e^{\beta_{n}+\beta_{n-1}+\cdots+\beta_{0}} x_{0} \\
& \leqslant e^{\sum_{n=0}^{\infty} \beta_{n}} x_{0} \\
& <\infty
\end{aligned}
$$

which implies that $x_{n}$ is bounded. Since $\sum_{n=0}^{\infty} \alpha_{n}<\infty$, then $\sum_{n=0}^{\infty} \beta_{n}<\infty$. So $\sum_{n=0}^{\infty} \beta_{n} x_{n}<\infty$. By Lemma 2.5, we know $\lim _{n \rightarrow \infty} x_{n}$ exists. It also shows that the $\lim _{n \rightarrow \infty}\left\|w_{n}-p\right\|$ exists for any $p \in \Gamma$.

(2) We show that the $\lim _{n \rightarrow \infty}\left\|u_{n}-p\right\|$ exists for any $p \in \Gamma$. 
By (3.6), we have

$$
\begin{aligned}
& \gamma\left(\alpha_{n}\left(k_{n}-1\right)+1\right)(2-2 \gamma / \lambda)\left\|G w_{n}\right\|^{2}+\alpha_{n}\left(1-\kappa-\alpha_{n}\right)\left\|T_{[n]}^{n} u_{n}-u_{n}\right\|^{2} \\
& \leqslant\left(\alpha_{n}\left(k_{n}-1\right)+1\right)\left\|w_{n}-p\right\|^{2}-\left\|w_{n+1}-p\right\|^{2}
\end{aligned}
$$

which implies that

$$
\lim _{n \rightarrow \infty}\left\|\mathrm{G} w_{n}\right\|=0
$$

and

$$
\lim _{n \rightarrow \infty} \alpha_{n}\left\|T_{[n]}^{n} u_{n}-u_{n}\right\|=0
$$

On one hand, by (3.2), we know

$$
\left\|w_{n+1}-p\right\|^{2} \leqslant\left\|u_{n}-p\right\|^{2}+2 \alpha_{n}\left\|u_{n}-p\right\|\left\|T_{[n]}^{n} u_{n}-u_{n}\right\|+\alpha_{n}^{2}\left\|T_{[n]}^{n} u_{n}-u_{n}\right\|^{2} .
$$

According to (3.9), we obtain

$$
\left\|w_{n+1}-p\right\| \leqslant\left\|u_{n}-p\right\| .
$$

On the other hand, by (3.5) and (3.8) we have

$$
\left\|u_{n}-p\right\| \leqslant\left\|w_{n}-p\right\| .
$$

From (3.10) and (3.11), we get

$$
\lim _{n \rightarrow \infty}\left\|w_{n}-p\right\|=\lim _{n \rightarrow \infty}\left\|u_{n}-p\right\|
$$

(3) We prove that

$$
\lim _{n \rightarrow \infty}\left\|w_{n+1}-w_{n}\right\|=0,
$$

and

$$
\lim _{n \rightarrow \infty}\left\|u_{n+1}-u_{n}\right\|=0
$$

In fact, it follows from (3.1) that

$$
\begin{aligned}
\left\|w_{n+1}-w_{n}\right\|^{2} & =\left\|P_{S}\left[\left(1-\alpha_{n}\right) u_{n}+\alpha_{n} T_{[n]}^{n} u_{n}\right]-w_{n}\right\|^{2} \\
& =\left\|P_{S}\left[\left(1-\alpha_{n}\right) u_{n}+\alpha_{n} T_{[n]}^{n} u_{n}\right]-P_{S} w_{n}\right\|^{2} \\
& \leqslant\left\|\alpha_{n}\left(T_{[n]}^{n} u_{n}-u_{n}\right)+\left(u_{n}-w_{n}\right)\right\|^{2} \\
& \leqslant 2 \alpha_{n}^{2}\left\|T_{[n]}^{n} u_{n}-u_{n}\right\|^{2}+2\left\|u_{n}-w_{n}\right\|^{2} \\
& =2 \alpha_{n}^{2}\left\|T_{[n]}^{n} u_{n}-u_{n}\right\|^{2}+2 \gamma^{2}\left\|G^{*} G w_{n}\right\|^{2} \\
& =2 \alpha_{n}^{2}\left\|T_{[n]}^{n} u_{n}-u_{n}\right\|^{2}+2 \gamma^{2}\left\langle G^{*} G w_{n}, G^{*} G w_{n}\right\rangle \\
& =2 \alpha_{n}^{2}\left\|T_{[n]}^{n} u_{n}-u_{n}\right\|^{2}+2 \gamma^{2}\left\langle G w_{n}, G G^{*} G w_{n}\right\rangle \\
& \leqslant 2 \alpha_{n}^{2}\left\|T_{[n]}^{n} u_{n}-u_{n}\right\|^{2}+\left(4 \gamma^{2} / \lambda\right)\left\|G w_{n}\right\|^{2} \\
& =2\left(\alpha_{n}\left\|T_{[n]}^{n} u_{n}-u_{n}\right\|\right)^{2}+\left(4 \gamma^{2} / \lambda\right)\left\|G w_{n}\right\|^{2} .
\end{aligned}
$$

This together with (3.8) and (3.9) imply that

$$
\lim _{n \rightarrow \infty}\left\|w_{n+1}-w_{n}\right\|=0 .
$$

Similarly, it follows from (3.1) and (3.12) that

$$
\begin{aligned}
\left\|u_{n+1}-u_{n}\right\|^{2} & =\left\|\left(I-\gamma G^{*} G\right) w_{n+1}-\left(I-\gamma G^{*} G\right) w_{n}\right\|^{2} \\
& \leqslant 2\left\|w_{n+1}-w_{n}\right\|^{2}+2\left\|\gamma G^{*} G\left(w_{n+1}-w_{n}\right)\right\|^{2} \\
& \leqslant 2\left\|w_{n+1}-w_{n}\right\|^{2}+\left(8 \gamma^{2} / \lambda^{2}\right)\left\|w_{n+1}-w_{n}\right\|^{2} \\
& =2\left(1+4 \gamma^{2} / \lambda^{2}\right)\left\|w_{n+1}-w_{n}\right\|^{2} \\
& \rightarrow 0(\text { as } n \rightarrow \infty) .
\end{aligned}
$$


(4) In step (1), we have known $\left\{w_{n}\right\}$ is a bounded sequence. Let $w^{*}$ be a weak cluster point of $\left\{w_{n}\right\}$, there exists a sequence $\left\{w_{n_{k}}\right\}$ such that $\left\{w_{n_{k}}\right\}$ converges weakly to $w^{*}$, then $G w^{*}=0$. It follows that $w^{*} \in \operatorname{Fix}\left(I-\gamma G^{*} G\right)$. On the other hand, when $n \geqslant 2, w_{n}=P_{S} w_{n}$, as $w_{n_{k}}$ converges weakly to $w^{*}$, we obtain that $\mathrm{P}_{\mathrm{S}} w^{*}=w^{*}$, that is to say $w^{*} \in \operatorname{Fix}\left(\mathrm{P}_{\mathrm{S}}\right)$.

Therefore $w^{*} \in \operatorname{Fix}\left(P_{S}\right) \bigcap \operatorname{Fix}\left(I-\gamma G^{*} G\right)$.

(5) Next we prove that $w_{n} \rightarrow w^{*}$.

By contradiction, without loss of generality, we assume that there exists another subsequence $w_{n_{l}} \subset$ $w_{n}$.

Case 1. If $w_{n_{l}}$ is a convergent sequence, then $w_{n_{l}} \rightarrow w^{* *}$, with $w^{*} \neq w^{* *}$. Otherwise, Case 2, the sequence $w_{n_{l}}$ has at least two subsequences not convergent to the same point. We assume $w_{n_{l_{k}}} \rightarrow w_{*}$, with $w^{*} \neq w_{*}$. Consequently, by the existence of $\lim _{n \rightarrow \infty}\left\|w_{n}-p\right\|$ and the Opial property, in Case 1, we have

$$
\begin{aligned}
\liminf _{n_{i} \rightarrow \infty}\left\|w_{n_{i}}-w^{*}\right\| & <\liminf _{n_{i} \rightarrow \infty}\left\|w_{n_{i}}-w^{* *}\right\| \\
& =\liminf _{n \rightarrow \infty}\left\|w_{n}-w^{* *}\right\| \\
& =\liminf _{n_{j} \rightarrow \infty}\left\|w_{n_{j}}-w^{* *}\right\| \\
& <\liminf _{n_{j} \rightarrow \infty}\left\|w_{n_{j}}-w^{*}\right\| \\
& =\liminf _{n \rightarrow \infty}\left\|w_{n}-w^{*}\right\| \\
& =\liminf _{n_{i} \rightarrow \infty}\left\|w_{n_{i}}-w^{*}\right\| .
\end{aligned}
$$

This is a contradiction, then $w^{*}=w^{* *}$. In Case 2, the same method as above, we have $w^{*}=w_{*}$. Therefore $w_{n} \rightarrow w^{*}$. This completes the proof.

\section{Numerical example for SEP in $R^{2}$}

In this section, we consider split equality problem for some special cases of $\mathrm{k}$-asymptotically strictly pseudo-contraction mapping in Definition 2.2, $\mathrm{H}_{1}=\mathrm{H}_{2}=\mathrm{H}_{3}=\mathrm{R}^{2}$.

$\mathrm{T}_{i}, i=1,2$ are two $\mathrm{k}$-asymptotically strictly pseudo-contraction mappings $(\mathrm{k}(\mathrm{i})=0)$,

$$
\begin{gathered}
\mathrm{T}_{1}=\left[\begin{array}{cc}
\sqrt{2} / 2 & \sqrt{2} / 2 \\
-\sqrt{2} / 2 & \sqrt{2} / 2
\end{array}\right], \quad \mathrm{T}_{2}=\left[\begin{array}{cc}
\sqrt{3} / 2 & 1 / 2 \\
-1 / 2 & \sqrt{3} / 2
\end{array}\right], \\
\left\|\mathrm{T}_{i} x-\mathrm{T}_{i} \mathrm{y}\right\|^{2} \leqslant\|x-y\|^{2}+\kappa_{i}\left\|x-\mathrm{T}_{i}^{n} x-\left(y-\mathrm{T}_{i}^{n} \mathrm{y}\right)\right\|^{2},
\end{gathered}
$$

for all $x, y \in D(T)$. Here $k_{n} \equiv 1, k=0, i=1,2$.

$\mathrm{C}=\bigcap_{i=1}^{2} \operatorname{Fix}\left(\mathrm{T}_{i}\right), \mathrm{Q}=[-1,1 ;-1,1], \mathrm{S}=\mathrm{C} \times \mathrm{Q}, \mathrm{H}=\mathrm{H}_{1} \times \mathrm{H}_{2}$, we put $\mathrm{T}_{\mathrm{i}} \oplus \mathrm{I}, \mathrm{i}=1,2$ still marked as $\mathrm{T}_{i}, i=1,2$,

$$
\mathrm{T}_{1}=\left[\begin{array}{cccc}
\sqrt{2} / 2 & \sqrt{2} / 2 & 0 & 0 \\
-\sqrt{2} / 2 & \sqrt{2} / 2 & 0 & 0 \\
0 & 0 & 1 & 0 \\
& & 0 & 1
\end{array}\right], \quad \mathrm{T}_{2}=\left[\begin{array}{cccc}
\sqrt{3} / 2 & 1 / 2 & 0 & 0 \\
-1 / 2 & \sqrt{3} / 2 & 0 & 0 \\
0 & 0 & 1 & 0 \\
0 & 0 & 0 & 1
\end{array}\right]
$$

Let

$$
\mathrm{A}=\left[\begin{array}{ll}
1 & 2 \\
3 & 4
\end{array}\right], \mathrm{B}=\left[\begin{array}{ll}
2 & 3 \\
1 & 4
\end{array}\right]
$$

Let $P_{s}\left\{x_{1}, x_{2}, y_{1}, y_{2}\right\}=\left\{0,0, y_{1}, y_{2}\right\},\left\{y_{1}, y_{2}\right\} \in Q,\left\{w_{n}\right\}$ be generated by

$$
w_{1}=\{0,0,0.5,0.5\} \in H, \quad u_{n}=\left(I-\gamma G^{*} G\right) w_{n} w_{n+1}=P_{S}\left[\left(1-\alpha_{n}\right) u_{n}+\alpha_{n} T_{[n]}^{n} u_{n}\right] .
$$


Here $[\mathrm{n}]=\mathrm{n} \bmod 2$,

$$
\begin{gathered}
G=\left[\begin{array}{cccc}
10 & 14 & -5 & -15 \\
14 & 20 & -8 & -22 \\
-5 & -8 & 5 & 10 \\
-15 & -22 & 10 & 25
\end{array}\right], G^{*}=\left[\begin{array}{cccc}
10 & 14 & -5 & -15 \\
14 & 20 & -8 & -22 \\
-5 & -8 & 5 & 10 \\
-15 & -22 & 10 & 25
\end{array}\right], \\
G^{*} G=\left[\begin{array}{cccc}
546 & 790 & -337 & -883 \\
790 & 1144 & -490 & -1280 \\
-337 & -490 & 214 & 551 \\
-883 & -1280 & 551 & 1434
\end{array}\right] .
\end{gathered}
$$

Let $0<\gamma=0.0001<2 / \rho\left(G^{*} G\right)=0.00060009, \alpha_{i}=\frac{1}{n^{100 / 99}}$ be the same as above assumptions. Here $\Gamma=\{0,0,0,0\}$,

$$
\lim _{n \rightarrow \infty} w_{n}=\{0,0,0,0\} \in \Gamma \text {. }
$$

Table 1: Initial point $\{0,0,0.5,0.5\}$.

\begin{tabular}{ccccc}
\hline Initial point & $w_{2 n+1}$ & Iter & Time & error \\
\hline$\{0,0,0.5,0.5\}$ & $\{0,0,0.005,-0.0019\}$ & $2 * 10000+2$ & 0.554 & 0.0054 \\
$\{0,0,0.5,0.5\}$ & $\left\{0,0,0.6844 * 10^{-3},-0.2633 * 10^{-3}\right\}$ & $2 * 15000+2$ & 0.818 & $7.3333 * 10^{-4}$ \\
$\{0,0,0.5,0.5\}$ & $\left\{0,0,0.9357 * 10^{-4},-0.36 * 10^{-4}\right\}$ & $2 * 20000+2$ & 1.080 & $1.0026 * 10^{-4}$ \\
$\{0,0,0.5,0.5\}$ & $\left\{0,0,0.1749 * 10^{-5},-0.0673 * 10^{-5}\right\}$ & $2 * 30000+2$ & 1.63 & $1.8739 * 10^{-6}$ \\
$\{0,0,0.5,0.5\}$ & $\left\{0,0,0.3269 * 10^{-7},-0.1258 * 10^{-7}\right\}$ & $2 * 40000+2$ & 2.181 & $3.5025 * 10^{-8}$ \\
\hline
\end{tabular}

\section{Acknowledgment}

We wish to thank the referees for their helpful comments and suggestions. This research was supported by NSFC Grant (No. 11071279) and National Science Foundation for Young Scientists of China (No. 11301379) and also by Tianjin Natural Science Foundation (14JCYBJC17600).

\section{References}

[1] V. Berinde, Iterative Approximation of Fixed Points, Editura Efemeride, Baia Mare, (2002). 2.5

[2] F. E. Browder, W. V. Petryshyn, Construction of fixed points of nonlinear mappings in Hilbert spaces, J. Math. Anal. Appl., 20 (1967), 197-228. 2.2

[3] C. Byrne, A unified treatment of some iterative algorithms in signal processing and image reconstruction, Inverse Problems, 20 (2004), 103-120. 1

[4] Y. Censor, T. Bortfeld, B. Martin, A. Trofimov, A unified approach for inversion problems in intensity-modulated therapy, Phys. Medicine Biol., 51 (2006), 2353-2365. 1

[5] S.-S. Chang, Y. J. Cho, J. K. Kim, W. B. Zhang, L. Yang, Multiple-set split feasibilty problems for asymptotically strict pseudocontractions, Abstr. Appl. Anal., 2012 (2012), 12 pages. 2.2

[6] R. D. Chen, J. Wang, H. Zhang, General split equality problems in Hilbert space, Fixed Point Theory Appl., 2014 (2014), 8 pages. 2.6

[7] A. Moudafi, A relaxed alternating CQ-algorithm for convex feasibility problems, Nonlinear Anal. Theory Meth. Appl., 79 (2013), 117-121. 1

[8] A. Moudafi, Alternating CQ-algorithm for convex feasibility and split fixed point problems, J. Nonlinear Convex Anal., 15 (2014) 809-818. 1, 1

[9] J. Quan, S.-S. Chang, Multiple-set split feasibility problems for K-asymptotically strictly pseudo-nonspreading mapping in Hilbert space, J. Inequal. Appl., 2014 (2014), 14 pages. 1, 2.2, 2.4

[10] L. Shi, R. D. Chen, Y. J. Wu, An iterative algorithm for the split equality and multiple-sets split equality problem, Abstr. Appl. Anal., 2014 (2014). 2.7 